\title{
Minimum-Energy Broadcast Using Practical Directional Antennas in All-Wireless Networks
}

\author{
Sabyasachi Roy, Y. Charlie Hu, and Dimitrios Peroulis \\ School of Electrical and Computer Engineering \\ Center for Wireless Systems and Applications \\ Purdue University \\ West Lafayette, IN 47907 \\ Email: $\{$ roy0,ychu,dperouli\}@purdue.edu
}

\author{
Xiang-Yang Li \\ Department of Computer Science \\ Illinois Institute of Technology \\ Chicago, IL, 60616 \\ Email: xli@cs.iit.edu
}

\begin{abstract}
Energy-efficient broadcast communication is an important problem in wireless ad hoc networks. Previously, minimum-energy broadcast that exploits the broadcast nature of radio transmission has been studied and shown to be NP-complete for omnidirectional antennas. In this paper, we investigate the minimum-energy broadcast problem under a wide spectrum of directional antenna models, including sectored antennas with fixed sectors and beamwidth and antenna array-based smart antennas with varying degrees of beam orientation and beamwidth We first propose the RF design and implementation of each model, which suggests the practical parameters of antennas under that model. We then show that the minimum-energy broadcast problem under each of the antenna models is NP-complete. Lastly, we present a heuristic algorithm based on BIP for the above problem under each directional antenna model and experimentally compare the energy efficiency of using different directional antennas using these heuristics. Our results show that using antennas with adjustable orientation and variable beamwidth gives the best results. For such antennas, the scanning angle is the dominant factor in improving the quality of the broadcast trees. The number of antennas per node is not that critical to obtaining a better broadcast tree as long as it is large enough to cover the entire $360^{\circ}$ around a node so as to prevent network partitioning.
\end{abstract}

\section{INTRODUCTION}

A wireless ad hoc network consists of a collection of wireless nodes dynamically forming a temporary network without the use of any existing network infrastructure or centralized administration. In such a network, nodes operate both as hosts and routers, forwarding packets for other nodes that may not be within direct transmission range of each other. Such decentralized networks can enable flexible, infrastructure-less and robust data and service access to support ubiquitous computing environments. Typically, each node in such a network has limited energy resources. Consequently, energy efficiency is an important design consideration for such networks.

In many deployment scenarios, broadcast communication provides a bandwidth-efficient mechanism to communicate information between a source and a group of nodes. Broadcast can be used for data dissemination from a single data source, coordination and control among the nodes in the network, resource discovery and as a primitive operation in on-demand unicast protocols such as DSR [15] and AODV [23].

An important problem in broadcast is to adjust the transmission power of individual nodes so as to minimize the overall transmission power in the entire network. This is known as the minimumenergy broadcast problem. There have been several studies on the minimum-energy broadcast problem in wireless ad hoc networks using omnidirectional antennas. In particular, the problem has been shown to be NP-complete [8], [18], [2] and several heuristics have been proposed [30], [2].

Using omnidirectional antennas in wireless ad hoc networks can be highly inefficient in terms of power and capacity because a very small portion of the transmitted power is actually intercepted by the antenna of the intended receiver. The rest of the power spreads in the surrounding space causing unwanted and harmful interference to other users. Consequently, transmission and reception of information through directional antennas is highly desirable. A directional antenna can be viewed in general as a spatial filter that confines the radiated energy into a small volume appropriate for the intended user.

This spatial filtering leads to numerous important advantages, particularly for 802.11-type systems [3], [28]. First, multipath-based fading, which is particularly harmful in urban environment and indoor communications, is considerably reduced. Second, for a given power level the communication range of each node is significantly increased because of the high directive gain of the antenna. Alternatively, a given range can be covered with dramatically reduced transmitted power, which leads to important savings in battery life. This advantage has the immediate consequence of improving the energy efficiency of broadcast in wireless ad hoc networks. Third, higher data rates and throughput are possible due to the improved sensitivity of the receiver. For instance, an $802.11 \mathrm{~b}$ user transmitting at 1 to 2 Mbps with an omnidirectional antenna, can successfully switch to 11 Mbps through a high-gain directional antenna [3]. Even more significant benefits are possible in $802.11 \mathrm{a} / \mathrm{g}$. Finally, interference between two (or more) adjacent users can be practically eliminated by proper positioning of their antennas.

Directional antennas in operation are characterized by the beam orientation and beamwidth and by definition cannot connect all neighboring nodes that may be spread over the entire space (0$360^{\circ}$ for two-dimensional (2-D) communication models). As a result, adaptive (frequently called smart) directional antennas are required to resolve this issue in applications that involve mobile users or changing traffic patterns. There are a variety of techniques to achieve this and different techniques can lead to different practical beam orientation and beamwidth.

In this paper, we investigate the minimum-energy broadcast problem using practical directional antennas. We consider a wide spectrum of directional antenna models, including both sectored antennas which have fixed sectors and beamwidth and antenna array-based smart antennas which have varying degrees of design freedom in terms of the beam orientation and the beamwidth. Our major contributions in this paper are as follows. First, we propose the RF design and implementation of each array-based directional antenna model (Section IV) which determines the practical parameters of the antennas under each model. Second, we show that the minimumenergy broadcast problem under each of the antenna model is NPcomplete (Section V), present several methods with certain provable performances, and experimentally compare the energy efficiencies of 


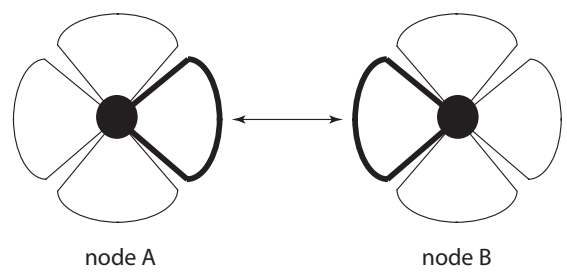

Fig. 1. Sectored Antennas (Figure reproduced from [3].) Bold lines indicate the active sectors that enable the communication between nodes $\mathrm{A}$ and $\mathrm{B}$.

the heuristic algorithms using different types of directional antennas as well as of the one that uses omnidirectional antennas (Section VI).

\section{RELATED WORK}

The problem of minimizing the energy consumption in wireless networks has received significant attention over the last few years [4], [33], [30], [5], [34], [29], [35], [17], [14], [22]. The minimumenergy broadcast problem in wireless ad hoc networks was first introduced by Wieselthier et al. in [30]. Since then, there have been a number of studies on the complexity of the problem and on the design of heuristic algorithms [29], [8], [10]. However, all of these studies focused on heuristics assuming omnidirectional antennas. Wieselthier et al. in [32] for the first time proposed heuristics for finding minimum-energy broadcast trees with directional antennas. Two algorithms, namely, Directional BIP (D-BIP) and Reduced Beam BIP (RB-BIP), were introduced. However, both algorithms assume the existence of antennas capable of transmitting at any orientation and with arbitrary beamwidth above a certain threshold. An extension to BIP using sectored antenna (S-BIP) was presented in [19] in which the minimum incremental power is calculated on a per sector basis. The same can be applied to switched beam antennas as well. In [16], Kang and Poorvendran proposed Sectored Greedy Perimeter Broadcast efficiency (S-GPBE) for constructing minimum-energy broadcast trees with sectored antennas. S-GPBE works by adding one or more nodes in each iteration depending upon the greedy choice of maximizing broadcast efficiency, where broadcast efficiency is defined as the amount of incremental energy spent per node added. In [12], a constraint formulation in terms of mixed integer linear programming was given for the minimum-energy multicast problem using directional antennas. This work assumes each node is provided with a single directional antenna with a fixed beam width and arbitrarily adjustable orientation. In summary, previous work on minimumenergy broadcast have considered either only sectored antennas or antenna array-based smart antennas with arbitrary orientation and beam width. Our work differs from these works by considering a wide spectrum of smart antennas with practical parameters from actual RF design and implementation.

\section{A Classification of Adaptive Directional Antennas}

Two major types of practical adaptive antennas are available today. The first type is commonly referred to as sectored antennas [1], [3] and it includes a number of fixed sectors that can provide full coverage in azimuth (Figure 1). When sectored antennas are employed, each network node is connected to $N$ antennas (each sector has an azimuthal bandwidth of $360^{\circ} / N$ ). Each node has the capability to dynamically choose the sector needed to communicate with another node as shown in Figure 1. This conceptually simple scheme of dynamic reconfiguration requires one (or more) inexpensive passive antenna elements per sector and a network of switches that control the selection of the antennas. Additionally, it needs an adaptive algorithm, computational power and digital signal processing to be implemented.
Although 10-15 years ago these requirements might have implied a high cost, this is not a true limitation today [3].

The major advantages of the architecture of sectored antennas are the simplicity and relatively low cost required for their implementation. On the other hand, due to practical limitations, a sectored antenna cannot provide an arbitrarily small (azimuthal) beamwidth. Commercially available sectored antennas are typically designed for beamwidths of $180,120,90,60$ and $45^{\circ}$. Since each sector typically requires one antenna (with a ground plane), a large number of sectors would require an impractically high number of antennas.

The second type of smart antennas is based on the concept of antenna arrays. An antenna array primarily consists of $2 N+1$ ideal antenna elements (that are commonly omnidirectional antennas) spaced a fraction of the wavelength apart (Figure 2(a)). Typical distances between successive elements are $\lambda / 2$ and $\lambda / 4$, where $\lambda$ is the wavelength of the radiated wave. This spacing along with the phase difference of the current of each antenna element leads to preferred directions of radiation (radiation maxima) and directions with no radiated power (radiation minima). The design of an antenna array primarily consists of the selection of the topology (linear, planar, or conformal to a surface), number of elements $P=2 N+1$, interelement distance $d$ and excitation currents (amplitude and phase) to achieve a particular beamwidth, gain and side-lobe level (SLL). Although a low SLL is of minor importance in the transmitting mode, it becomes particularly meaningful in the receiving mode because it dominates the level of interference between adjacent nodes.

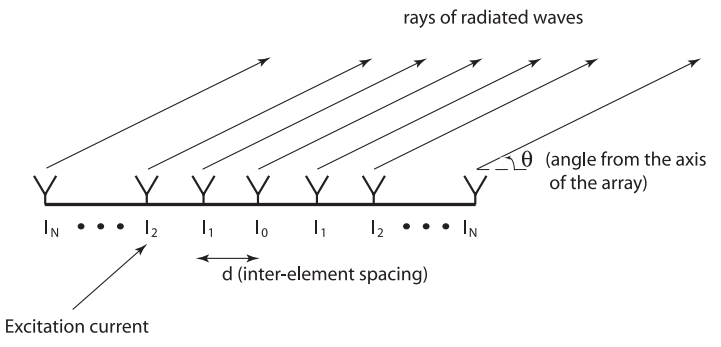

(a)
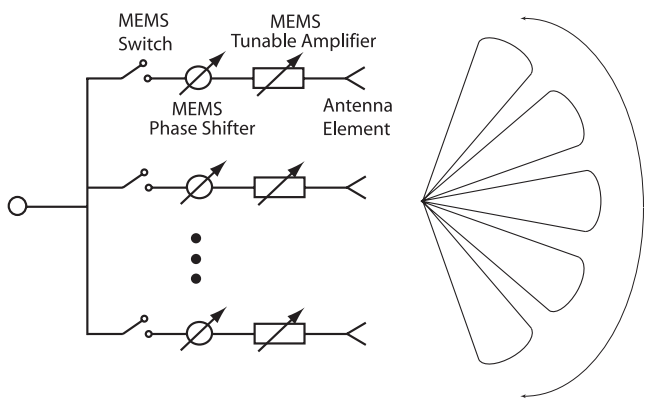

(b)

Fig. 2. (a) Antenna array block diagram and (b) implementation based on RF MEMS components. Notice the presence of the MEMS switches, tunable impedance networks and phase shifters used to tune the properties of the antennas.

Antenna arrays can be made adaptive by including tunable circuits before each antenna element (Figure 2(b)). For example, phased arrays (arrays that can tune the orientation of their main lobe) typically require one phase shifter per antenna element to control its relative phase. Additional reconfiguration may be achieved by other elements. These are discussed in detail in the following sections. 
While smart antenna arrays present a higher design complexity and cost than sectored antennas, their adaptive beam forming is very attractive in a number of applications. These antennas can dynamically optimize their gain for a desired direction and simultaneously reduce the interference in another one. Additionally, a wealth of information on algorithms for antenna arrays is already available [27].

\section{Practical Adaptive Directional Antennas}

In this section, we first present a taxonomy of directional antenna models. We then give the RF design and implementation of directional antennas with adjustable orientation and/or variable beamwidth using antenna arrays.

\section{A. Models of Adaptive Directional Antennas}

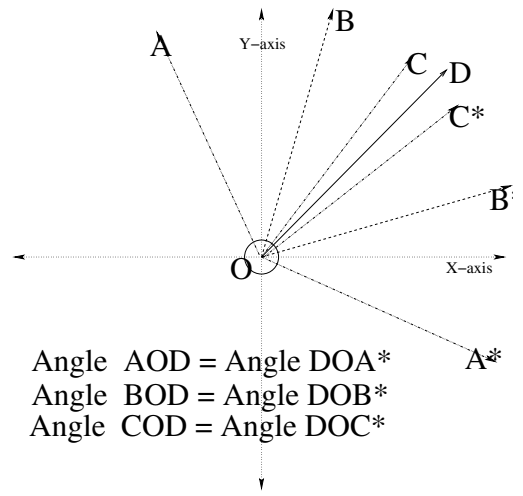

Fig. 3. Two parameters of a directional antenna: orientation and beamwidth.

Figure 3 shows a typical directional antenna. The vector $O D$ defines an orientation for an antenna. We denote this orientation vector as $\kappa . \angle A O A^{*}$ is the maximum possible beamwidth. We will denote this angle as $\alpha$. Similarly $\angle C O C^{*}$ is the minimum possible beamwidth and we will denote this as $\beta . \angle B O B^{*}$ is the angle at which a given antenna transmits at any given point. This is denoted by $\Theta$. Clearly $\beta \leq \Theta \leq \alpha$. $\alpha$ and $\beta$ are properties of the antenna and can not be changed. $\Theta$ is a variable and can be changed but it always ranges from a maximum of $\alpha$ and a minimum of $\beta$. Also note that the beamwidth can not be changed arbitrarily, i.e., the beamwidth changes in such a manner that it is symmetrical with respect to the orientation vector $\kappa$. Figure 3 shows that $\angle B O D=\angle B^{*} O D=\frac{\Theta}{2}$. $\kappa$ can or can not be changed depending upon the type of antenna we are considering. In this paper, we consider the following types of antennas.

1) Fixed orientation and fixed beamwidth (FOFB): This is the simplest antenna model in which an antenna can transmit at a given beamwidth and at a fixed orientation. While this is not an adaptive antenna, it is considered here for completeness of the presentation. In this type of antenna, the orientation vector $\kappa$ remains fixed once it is installed. Moreover, $\alpha=\beta$. Therefore, $\Theta$ is also fixed meaning that such an antenna can not change its beamwidth.

2) Fixed orientation and variable beamwidth (FOVB): In this model, each antenna has a fixed orientation $\kappa$, but has an adjustable beamwidth $\left[\kappa-\frac{\Theta}{2}, \kappa+\frac{\Theta}{2}\right]$. Again, the beamwidth should be within $\alpha$ and $\beta$.

3) Adjustable orientation and fixed beamwidth (AOFB): In this model, each antenna has an adjustable orientation $\kappa \in$ $\left[\kappa_{\min }, \kappa_{\max }\right]$, but the beamwidth is fixed, i.e., $\alpha=\beta$ and hence $\Theta$ is also fixed.
4) Adjustable orientation and variable beamwidth (AOVB): In this model, each antenna has an adjustable orientation $\kappa \in$ $\left[\kappa_{\min }, \kappa_{\max }\right]$, as well as an adjustable beamwidth $\left[\kappa-\frac{\Theta}{2}, \kappa+\frac{\Theta}{2}\right]$ for each orientation. Again, the beamwidth should be within $\alpha$ and $\beta$.

Although simulating the performance of a network can be achieved with arbitrary antenna models, practical and useful results are obtained only when realistic models are considered. Consequently, in the next section, we present some basic notes that justify the selection of these models, provide design details, discuss implementation challenges and limitations and briefly analyze the most promising available technologies for their fabrication.

\section{B. Directional Antenna Design}

The basic antenna design in all three cases is based on the DolphTshebyscheff arrays [7], [9]. A Dolph-Tshebysheff array is optimal in the sense that it provides the lowest side-lobe level for a given beamwidth. Alternatively, it provides the highest directivity for a given side-lobe level. Note that in our design, the beamwidth is defined as the beaming angle beyond which the gain drops by 3 $\mathrm{dB}$, as conventionally done in directional antenna design.

The design process for a fixed orientation and fixed beamwidth antenna is well-documented [7], [9] and will not be repeated here. We only mention that the basic design requirement is to determine the excitation currents of each antenna element for the specified orientation and beamwidth. In the following sections, we focus on the specific design details required to approximate the reconfigurable properties.

1) Directional Antenna Technology: RF MEMS: All of our designs follow the block diagram of the antenna array shown in Figure 2(b). This is a traditional phased array except for the inclusion of tunable Micro-Electro-Mechanical Systems (MEMS) that enable reconfiguration of the array. MEMS devices show remarkable advantages over their solid state counterparts (PIN diodes or FET transistors) that make them very attractive for many wireless applications. First, RF MEMS require very low DC power to operate (in the order of $\mu \mathrm{W}$ ) as compared to high-frequency diodes and transistors (in the order of $\mathrm{mW}$ ) [26]. Moreover, they are characterized by very small parasitics that lead to on-state insertion loss of less than $0.3 \mathrm{~dB}$ per device and isolation of higher than $30 \mathrm{~dB}$ per device even up to $100 \mathrm{GHz}$. Furthermore, they are much more linear than solid state devices, since they are completely passive components with no semiconductor junctions. This improves the inter modulation distortion by approximately $30 \mathrm{~dB}$. Note that while MEMS' reliability is still a technology under research and development, several MEMS devices have already demonstrated impressive repeatability of over 100 billion cycles [25]. Since no other technology has been able to match this RF performance, the RF MEMS' potential is very promising in a number of commercial (tunable filters, phase shifters, wireless telecommunications switches) and defense (phased arrays, high-performance matching networks) applications. A number of companies are currently pursuing this technology (Agilent, Radant MEMS, Teravicta, Xcom Wireless, EMAG Technologies, Magfusion and others) and commercial products are expected to become available in the next few years.

In particular, our reconfigurable antenna design is based on the properties of previously developed MEMS switches [24], tunable amplifiers [20] and phase shifters [26] (Figure 2). MEMS switches enable us to include or exclude an antenna element in the array after the array has been fabricated. This will allow us to tune the array beamwidth. The phase shifters are needed to adjust the relative 
phase of each component and achieve a tunable orientation. Finally, the tunable power amplifiers control the current amplitude of each antenna element, which results in a tunable taper in the array. As shown in Section IV-B.3, this property can be exploited to assure a nearly constant beamwidth for adjustable orientations. The details for the role of each MEMS subsystem are presented in the following designs.

2) Fixed Orientation and Variable Beamwidth Array Design:

This is the simplest of the proposed designs and requires only the MEMS switches and tunable amplifiers shown in Figure 2. The basic idea is to achieve an adjustable beamwidth by controlling the number of elements in the antenna array. Sharp beams require a large number of elements, while few elements are typically sufficient for broad beams. MEMS switches are ideal components for selecting/deselecting antenna elements due to their ultra low-loss, high-isolation and low parasitics. The antenna elements that will be part of the array for a specific beamwidth will be called active elements. The currents for the inactive elements will be assumed to be zero (non-idealities arising from mutual antenna coupling will be considered in a future investigation). Besides MEMS switches, tunable amplifiers are also necessary in this design to adjust the excitation currents of the active elements for each beamwidth. This assures a constant side-lobe level (SLL) for all cases.

This design starts by considering a traditional broadside (since no adjustable orientation is needed) Dolph-Tshebyscheff array. To demonstrate the process we will employ an example of a 15-element antenna array $(P=2 N+1=15)$ with a SLL of $\mathrm{R}=26 \mathrm{~dB}(\mathrm{R}=$ $400)$ to assure low interference. The optimum inter-element spacing can be calculated by [1]

$$
d_{o p t}=\lambda\left[1-\frac{\cos ^{-1} \frac{1}{\gamma}}{\pi}\right]
$$

where $\gamma=\cosh \left[\frac{1}{P-1} \ln \left(R+\sqrt{R^{2}-1}\right)\right]$. This results in $d_{o p t}=$ $0.516 \lambda$, which for simplicity is rounded to $d=0.5 \lambda$ in the rest of the analysis. If all 15 elements are active, the desired relative current distribution is given by (from the center to the out most antenna element) $2.365: 2.311: 2.153: 1.908: 1.602: 1.265: 0.927:$ 1.000 , which results in a $3-\mathrm{dB}$ beamwidth of $7.9^{\circ}$. On the other hand, if 5 elements are active, the current distribution becomes 2.669 $: 2.112: 1.000: 0: 0: 0: 0: 0$ and the beamwidth changes to $25.4^{\circ}$. The need for switches and tunable amplifiers becomes obvious from this example. Table I summarizes all the results and shows that the achieved beamwidth tuning is about 5:1. ${ }^{1}$ Note that despite the adjustable beamwidth, a constant SLL of $26 \mathrm{~dB}$ is maintained in all designs. Further improvement is possible by adjusting the SLL below $26 \mathrm{~dB}$ for the 15-element array (such a technique is demonstrated in the following designs). This technique can be readily altered to achieve wider or narrower beamwidths depending on the total number of antenna elements in the array.

3) Adjustable Orientation and Fixed Beamwidth Array Design: This is a traditional phased-array antenna design that has already been proposed and implemented elsewhere [13], [21]. However, it is wellknown that the array beamwidth changes significantly as the main lobe is scanned far away from broadside. For instance, in the example of the previous section the beamwidth will be almost doubled (from 7.9 to $14.2^{\circ}$ ) when the array is scanned from 90 to $35^{\circ}$. If this broadening can be tolerated, a traditional phased-array design can

\footnotetext{
${ }^{1}$ The radiation patterns for all cases are shown in Figure 5 in the Appendix where the radiation pattern is normalized to $0 \mathrm{~dB}$.
}

TABLE I

Summarized results for the fixed orientation and adjustable beamwidth arrays. All arrays have a SLL of $26 d B$.

\begin{tabular}{|c|c|c|}
\hline \# Active elements & 3 -dB BW & Max/Min current \\
\hline 15 & $7.9^{\circ}$ & $2.551: 1$ \\
\hline 13 & $9.4^{\circ}$ & $2.543: 1$ \\
\hline 11 & $11.2^{\circ}$ & $2.724: 1$ \\
\hline 9 & $13.7^{\circ}$ & $2.878: 1$ \\
\hline 7 & $18.0^{\circ}$ & $2.925: 1$ \\
\hline 5 & $25.4^{\circ}$ & $2.669: 1$ \\
\hline 3 & $41.4^{\circ}$ & $1.809: 1$ \\
\hline
\end{tabular}

be implemented. However, if this broadening is not acceptable, the technique presented in this section partially alleviates the problem. The technique is effective for scanning angles $\pm 55^{\circ}$ from broadside, but it fails for angles close to end fire. A more complex array design is needed to cover these angles.

In the proposed array design, phase-shifters are required in addition to the switches and tunable amplifiers. The idea is to partially sacrifice the sharpness of the beam in broadside by limiting the number of active elements in the antenna. As the array is scanned away from broadside, more elements are added to increase its main lobe sharpness. Consequently, a nearly constant beamwidth can be achieved for relatively large scanning angles.

This technique is demonstrated with the same 15-element array shown in the previous section. In this design, it is assumed that the beamwidth needs to remain constant $\left( \pm 0.5^{\circ}\right)$ for as broad scanning as possible. The parameters for the design are given in Table II. ${ }^{2}$ It is interesting to note that not all orientations are implemented with the same SLL, but all of them remain under the threshold of $26 \mathrm{~dB}$. Reducing the SLL adds an additional degree of freedom in controlling the beamwidth.

TABLE II

Summarized results for the adjustable orientation and fixed beamwidth arrays. Symmetric results exist from 90 to $180^{\circ}$. The beamwidth (BW) for the conventional array is calculated assuming that all elements are active and the SLL is kept at $26 \mathrm{~dB}$.

\begin{tabular}{|c|c|c|c|}
\hline $\begin{array}{c}\text { Main lobe } \\
\text { angle }\left({ }^{\circ}\right)\end{array}$ & $\begin{array}{c}\text { \# Active } \\
\text { elements }\end{array}$ & $\begin{array}{c}\text { SLL } \\
{[\mathrm{dB}]}\end{array}$ & $\begin{array}{c}\text { BW } \\
\left(^{\circ}\right)\end{array}$ \\
\hline 35 & 15 & 26 & 14.2 \\
\hline 45 & 13 & 31 & 14.2 \\
\hline 55 & 11 & 28 & 14.0 \\
\hline 65 & 11 & 35 & 13.9 \\
\hline 75 & 9 & 26 & 14.2 \\
\hline 85 & 9 & 28 & 14.2 \\
\hline 90 & 9 & 26 & 14.1 \\
\hline
\end{tabular}

4) Adjustable Orientation and Variable Beamwidth Array Design: This design is primarily a combination of the previous two designs. All adjustable MEMS components (switches, phase shifters and tunable amplifiers) are required for this antenna array. The limitations mentioned in the previous section for scanning angles less than $30^{\circ}$ and greater than $150^{\circ}$ also apply in this case. In addition, further practical limitations apply as the following discussion shows.

Table III presents an illustrative example of the attempt to tune the beamwidth at scanning angles of $60^{\circ}$ by utilizing the technique

${ }^{2}$ The simulated radiation pattern for these parameters are shown in Figure 6 in the Appendix. 
TABLE III

Summarized results for the adjustable-orientation, adjustable-beamwidth arrays. The beamwidth tuning for large scanning angles is limited by the requirement of maintaining the SLL below $26 d B$ (compare with Table I).

\begin{tabular}{|c|c|c|}
\hline Scanning angle $=60^{\circ}$ & & \\
\hline \hline \# active elements & 3-dB BW $\left(^{\circ}\right)$ & SLL [dB] \\
\hline 15 & 9.4 & 26 \\
\hline 13 & 10.8 & 26 \\
\hline 11 & 13.0 & 26 \\
\hline 9 & 16.0 & 26 \\
\hline 7 & 20.9 & 26 \\
\hline 5 & 29.7 & 22.5 \\
\hline 3 & 50.0 & 7 \\
\hline
\end{tabular}

introduced in Section IV-B.2. ${ }^{3}$ It shows that under the limitation of accepting a SLL of less than $26 \mathrm{~dB}$, not all element combinations can be accepted. For instance, at $60^{\circ}$ it is not possible to use only 3 elements since the SLL is unacceptably high. As a result, the beamwidth tuning range at this angle is limited to $2.23: 1$, or $3.16: 1$ if an SLL of $22.5 \mathrm{~dB}$ can be accepted. This beamwidth tuning sensitivity is increased for larger scanning angles and therefore the tuning range will be decreased. Mechanical steering can be utilized to alleviate this limitation. Alternatively, planar arrays (instead of linear ones) can significantly improve this performance as well.

\section{ENERGY EFFICIENT BROADCAST WITH DIRECTIONAL ANTENNAS}

In this section, we first state the problem of minimum-energy broadcast using generic directional antennas. We then prove that this problem is NP-complete under different directional antenna models. We will then present several broadcast methods with provable performances. We first define the minimum-energy broadcast with directional antennas as follows.

Definition 1 (Directional-Antenna Min-Energy Broadcast (DAMEB)): Assume that we are given a set $V$ of nodes $\left\{v_{1}, v_{2}, \cdots, v_{n}\right\}$ with each node $v_{i}$ equipped with $n_{i}$ directional antennas $\left[a_{1}, a_{2}, \cdots, a_{n_{i}}\right]$ capable of transmitting at any power level, a source node $S$ and a non-negative number $B$. The decision version of the directionalantenna minimum-energy broadcast problem is to decide if there is a power assignment to each antenna of each node such that the induced communication graph spans all nodes and the total power cost of broadcast is at most $B$ ? The antennas behave as described in Section IV. The optimization version of the DAMEB is to find a broadcast tree rooted at the source node $S$ with the minimum total power.

We assume that the power needed to support the communication from node $v_{i}$ to node $v_{j}$ is proportional to $\left\|v_{i} v_{j}\right\|^{2}$, the beamwidth (denoted as $\Theta_{i, j}$ ) of node $v_{i}$ 's sector containing node $v_{j}$, and the beamwidth (denoted as $\Theta_{j, i}$ ) of node $v_{j}$ 's sector containing node $v_{i}$. Here $\left\|v_{i} v_{j}\right\|$ is the Euclidean distance between nodes $v_{i}$ and $v_{j}$. We also assume that the communication is reliable, i.e., no energy is consumed for retransmitting packets. If there are multiple downstream receiving nodes lying inside a sector of sending node $v_{i}$, the power needed by node $v_{i}$ to send data to all these downstream receiving nodes is the minimum transmission power needed to reach all these nodes. The total power needed by a node $v_{i}$ is the total power needed by all its $n_{i}$ antennas. Notice that it is possible that some of its antennas do not have downstream receiving nodes in a broadcast tree, and thus that specific antennas will not consume power. Remember

\footnotetext{
${ }^{3}$ The simulated radiation patterns are shown in Figure 7 in the Appendix.
}

that there are four different possible antennas: fixed orientation and variable beamwidth (FOVB), fixed orientation and fixed beamwidth (FOFB), adjustable orientation and fixed beamwidth (AOFB) and adjustable orientation and variable beamwidth (AOVB). Thus, $\Theta_{i, j}$ and $\Theta_{j, i}$ are fixed values for antennas of FOFB and AOFB, and they are adjustable for antennas of FOVB and AOVB.

For FOFB antennas, we can only adjust the transmission power of each antenna. Given a power assignment $p_{i}$ for each node $v_{i}$, the induced communication graph has a directed link $\left(v_{i}, v_{j}\right)$ if $v_{i}$ and $v_{j}$ are inside the active directed sectors of each other. It is wellknown that the minimum-energy broadcast problem is NP-hard when omnidirectional antenna is used. In the next section, we show that the minimum-energy broadcast with directional antennas is still NP-hard.

\section{A. Fixed Orientation and Fixed Beamwidth}

We first show that the minimum-energy broadcast with fixed orientation and fixed beamwidth (FOFB) is NP-complete.

Theorem 1: The problem of finding a minimum-energy broadcast tree with each node having fixed orientation and fixed beamwidth antennas is NP-complete.

See appendix for the proof.

\section{B. Adjustable Orientation and Fixed Beamwidth}

We now prove that the minimum-energy broadcast problem is NPcomplete with adjustable orientation and fixed beamwidth antennas (AOFB). We first reformulate our problem into a graph theoretical problem. We have a graph $G^{\prime}=\left(V^{\prime}, E^{\prime}\right)$. There is an edge $\left(u^{\prime}, v^{\prime}, \kappa\right)$ with cost $c_{u^{\prime} v^{\prime}}$ if node $u^{\prime}$ can reach $v^{\prime}$ using an antenna at orientation $\kappa$ and transmitting at power $c_{u^{\prime} v^{\prime}}$. If at any given orientation and power, there are more than one nodes that can be reached then the power needed to reach all those nodes is the maximum of the power needed to reach all those nodes. The problem now is to find a power and orientation assignment to the nodes and their antennas such that all the nodes in the graph are covered and the total broadcast power is less than $B$. To show this, we give a reduction from the degree bounded minimum spanning tree (DBMST) problem [11] which is known to be NP-complete.

DBMST INSTANCE: Given a graph $G=(V, E)$ and a positive number $B$ and a positive integer $p<|V|$. Each edge $(u, v) \in E$ has a weight $w(u, v)$.

QUESTION: Is there a spanning tree with cost less than $B$ such that none of the nodes has degree greater than or equal to $p$.

Given an instance of the above DBMST problem, we perform the reduction as follows. We create another graph $G^{\prime}=\left(V^{\prime}, E^{\prime}\right)$ where $V^{\prime}=V \cup S$. Each node $v^{\prime} \in V^{\prime}$ can be thought of as a node having $p-1$ directional antenna with fixed beamwidth and variable orientation. For each edge $(u, v) \in E$ with cost $w(u, v)$, we have an edge $\left(u^{\prime}, v^{\prime}, \kappa_{u^{\prime} v^{\prime}}\right) \in E^{\prime}$ with cost $c_{u^{\prime} v^{\prime}}=w(u, v)$. Since $\kappa_{u^{\prime} v^{\prime}} \in$ $\left[0,360^{\circ}\right], \forall u^{\prime}, v^{\prime}$, we can choose infinite number of $\kappa_{u^{\prime} v^{\prime}}$ values and we choose a distinct value of $\kappa_{u^{\prime} v^{\prime}}$ for each ordered pair $\left(u^{\prime}, v^{\prime}\right)$. Now by choosing a small enough beamwidth we can ensure that an antenna of a node can focus on at most one node at any time. $S$ can be thought of as the root node. There are edges of the form $\left(S, u^{\prime}, \kappa_{S u^{\prime}}\right), \forall u^{\prime} \in V^{\prime}$ with $c_{S u^{\prime}}=C_{\max }$, where $C_{\max }$ is a value that is greater than $B$.

Theorem 2: The problem of finding a minimum-energy broadcast tree with each node having adjustable orientation and fixed beamwidth antenna is NP-complete.

Proof: We now prove that the above problem is NP-complete. Clearly the above problem is in NP because one can guess the orientations ( $\kappa$ values) and the powers of the antennas and check 
in polynomial time whether the total broadcast power is less than $B$ and whether all the nodes are reached. We continue by proving that a solution for the degree bounded spanning tree problem with the total cost less than $B$ implies a solution for the reduced problem with the total cost less than $B+C_{\max }$ and vice versa.

Suppose the degree bounded problem has a solution with a cost less than $B$. Suppose the set of edges in the solution are $E_{\text {sol }} \subseteq E$. For each edge $(u, v) \in E_{\text {sol }}$, we choose two corresponding edges $\left(u^{\prime}, v^{\prime}, \kappa_{u^{\prime} v^{\prime}}\right),\left(v^{\prime}, u^{\prime}, \kappa_{v^{\prime} u^{\prime}}\right) \in E^{\prime}$. Besides, we choose any one edge of the form $\left(S, u^{\prime}, \kappa_{S u^{\prime}}\right)$. We call the above set of chosen edges belonging to the set $E^{\prime}$ as $E^{\prime \prime}$. Now, starting from $S$ we do breadth first search to determine the outgoing and incoming edges for each node. During the breadth first search operation, we use edges in $E^{\prime \prime}$ only. If the breadth first search traversed from node $u^{\prime}$ to $v^{\prime}$, then we select the edge $\left(u^{\prime}, v^{\prime}, \kappa_{u^{\prime} v^{\prime}}\right)$ as an edge in our solution set $\left(E_{\text {sol }}^{\prime}\right)$. Note that except for $S$, each node will have at least one incoming edge. Since the degree bounded problem ensures that no edge has a degree more than $p$, we are assured that no node in the reduced problem uses more than $p-1$ antennas because out of the $p$ edges one corresponds to the incoming edge. At the end of the breadth first search, all the edges in $E^{\prime}$ sol give the solution for the problem of minimum-energy broadcast using AOFB antennas. These edges $\left(u^{\prime}, v^{\prime}, \kappa_{u^{\prime} v^{\prime}}\right)$ correspond to an orientation and power assignment to each antenna such that all nodes are covered and the total power is less than or equal to $B+C_{\max }$ with the source node $S$ transmitting at $C_{\max }$. Clearly, the cost of these edges is less than $B+C_{\max }$. The guarantee that all nodes are covered comes from the definition of the spanning tree problem. So, once we reach a node $v^{\prime} \in V^{\prime}-S$ through a transmission by $S$, we are guaranteed to reach all other nodes as well.

Now we prove the opposite. Suppose we have a solution for the reduced problem. Let the set of edges in the solution be $E_{\text {sol }}^{\prime} \subseteq E^{\prime}$. Now for a solution for the degree bounded problem, we choose edges $(u, v) \in E$ if $\left(u^{\prime}, v^{\prime}, \kappa_{u^{\prime} v^{\prime}}\right) \in E_{s o l}^{\prime}$. Since no node can transmit with more than $p-1$ antennas, the degree bound of $p$ is taken care of. Thus, it is easy to see that if the total cost of broadcast is less than $B+C_{\max }$, that implies that by choosing the edges as above we can get a degree bounded spanning tree with cost less than $B$. Also, since all the nodes are covered by the minimum-energy broadcast solution, the resulting tree spans all the nodes. One thing to note here is the value of $C_{\max }$ is chosen so that it is greater than $B$. This ensures that at most one outgoing edge from $S$ is chosen (corresponding to only one antenna transmitting). This is so because had the solution contained two edges then the total cost would have been greater than or equal to $2 \cdot C_{\max }$ which would have obviously been greater than or equal to $B+C_{\max }$. This would have contradicted our assumption of having a solution for the minimum-energy broadcast problem with total power less than $B+C_{\max }$. It is also important to note why we need one and only one outgoing edge from the root $S$. Consider the case when there are two edges emanating from $S$. It might be possible that the solution contains two different disjoint trees within the set of nodes $V^{\prime}-S$ such that $S$ acts as the bridging node. In such a case we do not get a solution for a spanning tree containing nodes in $V^{\prime}-S$ only. However, the constraint of $C_{\max } \geq B$ prevents such a case thereby ensuring that the proof works.

\section{Fixed Orientation and Variable Beamwidth}

Theorem 3: The problem of finding a minimum-energy broadcast tree with each node having fixed orientation and variable beamwidth antennas is NP-complete.

The NP-completeness of the case with directional antennas with fixed orientation and variable beamwidth follows from the NPcompleteness of the case with fixed orientation and fixed beamwidth. This is because the latter is a special case of the former.

\section{Adjustable Orientation and Variable Beamwidth}

Theorem 4: The problem of finding a minimum-energy broadcast tree with each node having adjustable orientation and variable beamwidth antennas is NP-complete.

The NP-completeness of the case with directional antennas with adjustable orientation and variable beamwidth follows from the NP-completeness of the case with adjustable orientation and fixed beamwidth, as the latter is a special case for the former.

\section{E. Minimum Energy Broadcast Algorithms For Directional Antennas}

We are now ready to design efficient broadcast protocols for directional antennas. We first review the BIP protocol for broadcast with omnidirectional antennas and some related algorithms developed from BIP. We then present how to extend these algorithms when using different types of directional antennas.

\section{1) Review of BIP-based Algorithms:}

a) BIP: The Broadcast Incremental Power (BIP) algorithm [30] is a centralized heuristic for the determination of a low power broadcast tree in networks with omnidirectional antennas. BIP is similar to Prim's Minimum Spanning Tree [6] algorithm. The only difference lies in the way edge weights are assigned. In Prim's algorithm, the edge weights are specified at the beginning and remain static during tree building. In BIP, the edge weights are adjusted dynamically after addition of each node. The edge weight $w_{i j}$ between two nodes $(i, j)$ is defined as $\Pi_{i j}-P_{i}$, where $P_{i}$ is the power at which node $i$ is transmitting in the partially built tree and $\Pi_{i j}$ is the power needed by node $i$ to reach $j$. In case, $\Pi_{i j}-P_{i}<0$, the edge is not considered, as a negative edge weight automatically means that the node $j$ is already covered by node $i$. After adding an edge $(i, j)$, weights $w_{i j}, \forall j$ are updated because $P_{i}$ changes. The algorithm terminates when all nodes have been covered. The complexity of the BIP algorithm is $O\left(n^{3}\right)$ as shown in [30].

b) RB-BIP: Reduced Beam BIP (RB-BIP) [32] extends BIP for the determination of a low power broadcast tree in networks with directional antennas. It first uses BIP to generate a low cost broadcast tree assuming the antennas are omnidirectional. After an initial tree is obtained, each transmitting antenna's beamwidth is reduced to the minimum possible value that provides coverage to all the downstream neighbors in the tree calculated by running BIP, subject to the constraint $\theta_{\text {min }} \leq \theta \leq 360$, where $\theta$ is the beamwidth after applying RB-BIP and $\theta_{\text {min }}$ is the lowest feasible value for the beamwidth. The complexity of RB-BIP is also the same as BIP, i.e., $O\left(n^{3}\right)$ as it uses BIP for the initial tree and then adjusts the beamwidth, which incurs an additional $O\left(n^{2}\right)$ complexity.

c) D-BIP: Directional BIP (D-BIP) [32] is another variant of the BIP algorithm for building low power broadcast trees in networks with directional antennas. While in the omnidirectional case, the incremental cost is only affected by the transmission power, in the directional case, the incremental cost is also affected by the beamwidth. Hence the weight of the edges between any two nodes $i$ and $j$ is of the form $w_{i j}^{\theta}$, i.e., it not only depends on the distance between the nodes but also the beamwidth at which node $i$ 's antenna is transmitting. Consequently, during each step of tree building, DBIP adds the node that requires the minimum incremental energy, which is calculated by varying the power level of transmission, the beamwidth, or both. Similar to BIP, after adding each node to the tree, the weights are readjusted and the algorithm terminates when all 
nodes have been covered. The complexity of D-BIP is also the same as BIP, i.e., $O\left(n^{3}\right)$ as it follows the same steps as BIP except that at each step it has the additional work of adjusting beamwidth to get the best incremental cost. This additional work is at most a constant times the work done in BIP and hence the $O\left(n^{3}\right)$ complexity. Note that in case when there are multiple antennas per node, the complexity becomes $O\left(p^{3} n^{3}\right)$, where $p$ is the number of antennas per node. In general, $p<<n$ and so the complexity varies as $O\left(n^{3}\right)$.

2) Our Method for Minimum Energy Broadcast With Adaptive Directional Antenna: We now propose our algorithms for minimumenergy broadcast with directional antennas. We use BIP, D-BIP, or an adapted version of RB-BIP (DRB-BIP) depending upon the type of antennas used, as shown in Table IV. In the following paragraph, we briefly describe the extended heuristics.

TABLE IV

Antenna types and the corresponding minimum-energy broadcast heuristic algorithms. $p$ is the number of antennas per node and $n$ is the number of nodes in the network.

\begin{tabular}{|l||c|c|c|c|c|}
\hline Ant. Type & Omni & Sectored & FOVB & AOFB & AOVB \\
\hline Algo. & BIP & DRB-BIP & D-BIP & D-BIP & D-BIP \\
\hline $\begin{array}{l}\text { Comple- } \\
\text { xity }\end{array}$ & $O\left(n^{3}\right)$ & $O\left(n^{3}\right)$ & $O\left(p^{3} n^{3}\right)$ & $O\left(p^{3} n^{3}\right)$ & $O\left(p^{3} n^{3}\right)$ \\
\hline
\end{tabular}

For omnidirectional antennas, we use the original BIP without any changes, which has a proven performance on the approximation ratio [29]. For sectored antenna, we use an extension of RB-BIP (DRB-BIP). After the initial tree is obtained using BIP, we find out all the downstream neighbors of a particular node. For each such downstream node, we find the sector that needs to transmit in order to reach that node. If there are more than one downstream neighbor nodes that should be reached by lighting up one sector, then the power at which that sector transmits is the minimum power needed to reach all such nodes. Those sectors that do not have any downstream neighbors in their range are turned off. This algorithm is called DRBBIP. If the power needed to support a link $(u, v)$ is proportional to $d_{u v}^{k}$ for some integer $k \geq 2$, where $d_{u v}$ is the distance between the nodes $u$ and $v$, we have the following theorem.

Theorem 5: Assume that the minimum beamwidth of the sectored antenna is $\alpha$. Then the power consumption of the structure constructed by DRB-BIP is at most $c \cdot 2 \pi / \alpha$ times of the optimum for fixed beamwidth antennas, where $c<12$ is the approximation ratio of the BIP algorithm for broadcast for omnidirectional antennas, and $\alpha$ is the beamwidth.

Proof: Let $\|T\|$ be the power consumption of a broadcast tree $T$ in a corresponding antenna model. Let $T$ be the tree constructed by the DRB-BIP method. Let $T_{D}$ be the tree used for broadcast with directional antennas; and $T_{O}$ be the tree used for broadcast with omnidirectional antennas. Similarly, we let $\mathrm{OPT}_{D}$ be the optimum broadcast tree with directional antennas; and $\mathrm{OPT}_{O}$ be the optimum broadcast tree with omnidirectional antennas. Remember that the power consumption of a sectored antenna with beamwidth $\alpha$ is $\alpha /(2 \pi)$-fraction of the power consumption of the corresponding antenna. Thus, $\frac{2 \pi}{\alpha}\left\|O P T_{D}\right\| \geq\left\|O P T_{O}\right\|$. Consequently, $\|T\| \leq$ $c\left\|O P T_{O}\right\| \leq \frac{2 c \pi}{\alpha}\left\|O P T_{D}\right\|$.

Similarly, for antennas with adjustable orientation and fixed beamwidth (AOFB), we have the following theorem.

Theorem 6: For AOFB antennas, the above DRB-BIP algorithm also implies a broadcast protocol whose total power consumption is no more than $c \cdot 2 \pi / \alpha$ times of the optimum.
We leave it as a future work to design broadcast protocols whose performance guarantee does not depend on the minimum beamwidth $\alpha$. For nodes with fixed orientation and variable beamwidth (FOVB), adjustable orientation and fixed beamwidth (AOFB) and adjustable orientation and variable beamwidth (AOVB), we use D-BIP. The procedure is as follows. We incrementally build the tree by adding one node at each step. The node added is the one that can be reached with the minimum incremental energy. To find such a node $j$, we find the minimum incremental energy needed for node $i(\forall i \neq j)$ to reach $j$, where $i$ is a node already in the partially built tree, which initially contains only the source node $s$. Thus node $i$ searches from a set of options available to it. These options depend on the type of antennas and the number of antennas. For example, a node with an FOVB antenna either increases its beamwidth or increases the transmission power or both. Similarly, a node with an AOFB antenna either changes its orientation or increases the transmission power or both, and a node with an AOVB antenna tries to change its orientation or increase its beamwidth or increase the transmission power or a certain combination of them. If a node has more than one antenna, it goes through all above steps for each antenna. Node $i$ changes its orientation, beamwidth, and the transmission power denoted by tuple $\left(\kappa_{1}, \Theta_{1}, P_{1}\right)$ to $\left(\kappa_{2}, \Theta_{2}, P_{2}\right)$ such that it reaches a new node and the incremental energy to do it is minimum. The node $i$ with the minimum such incremental power is chosen to reach a new node and the whole process is repeated until all the nodes are covered. The algorithm terminates either with a correct broadcast tree that spans all nodes or with a partial tree with no further addition of nodes possible. The latter case signifies a network partition.

Another method for finding the minimum-energy broadcast with directional antennas is as follows. Assume that the geometrical position of every wireless node is known. We first construct the Euclidean Minimum Spanning Tree (EMST) of all wireless nodes and then treat the EMST as a directed tree rooted at the source node $s$. For each internal node $u$, let $v_{1}, v_{2}, \cdots, v_{d}$ be its downstream nodes. We then find an optimum option of antennas for node $u$ to cover all its downstream nodes $v_{1}, v_{2}, \cdots, v_{d}$. Observe that this is possible since there are at most 6 downstream nodes for Euclidean minimum spanning tree. We call this method as D-MST. Similar to Theorem 5, we have the following theorem.

Theorem 7: Assume that the minimum beamwidth of the sectored antenna is $\alpha$. Then the power consumption of the structure constructed by D-MST is at most $c \cdot 2 \pi / \alpha$ times of the optimum for fixed beamwidth antennas, where $c<12$ is the approximation ratio of the EMST algorithm for broadcast for omnidirectional antennas, and $\alpha$ is the minimum beamwidth.

Note that we do not consider the D-MST heuristic for evaluation as MST has been shown in [31], [2], [30] not to perform well for minimum-energy broadcast problem because it fails to take advantage of the wireless broadcast advantage.

\section{PERformance Evaluation}

In this section, we experimentally compare the total broadcast power using the heuristic algorithms for different practical directional antennas.

\section{A. Propagation Model}

When considering uniform propagation conditions, the transmitted power decays as $R^{-\alpha}$, where $\alpha$ is a propagation constant and varies between 2 to 4 depending upon the communication medium. In our simulations we assume the transmission medium to be free space. We 
calculate the transmission powers using the equation given below.

$$
P_{R}=G_{R} G_{T}\left(\frac{\lambda}{4 \Pi R}\right)^{2} P_{T}
$$

In the above equation, $P_{R}$ is the received power. For our simulations, we used a receiving power threshold of $-80 \mathrm{dbm}$. $G_{T}$ and $G_{R}$ are the gains of the transmitting and receiving antennas, respectively. For our simulations, we assume that every node has an omnidirectional antenna for receiving. Since the power required to receive signals is much less than the power required to transmit, this is a reasonable assumption. $\lambda$ is the wavelength of the transmission. We use $\lambda=0.125 \mathrm{~m} . R$ is the distance of the receiver from the transmitter and we take the propagation constant to be 2 , i.e., the value used for free space. $P_{T}$ is the power of transmission. Note that the gain $G_{T}$ is a function of the beamwidth and is given by the following relation.

$$
G_{T}=\frac{30,000}{(\theta \cdot \Phi)}
$$

where $\theta$ is the horizontal beamwidth and $\Phi$ is the vertical beamwidth of the transmitting antenna. For all our simulations, we assume that all nodes are on a plane and hence we do not care about the vertical beamwidth and keep it at a constant value of $20^{\circ}$. The above relation does not hold true for large values of $\theta$. For omnidirectional antenna, the value of gain is 1 for receiving as well as transmitting.

\section{B. Methodology}

To study the impact of different types of antenna on the quality of the broadcast trees constructed, we simulated and compared the BIP-based algorithms as described in Section V-E.2 on networks with nodes having omnidirectional antennas, sectored antennas and directional (AOFB, FOVB and AOVB). Note that in our experiments, we do not consider FOFB antennas as they are the same as sectored antennas. We varied the network size between 10, 20, 50, 100 and 200. For each network size, 50 network instances were generated and simulated. For each instance, the nodes were randomly generated on a grid. For directional antennas their physical orientation was generated randomly. In scenarios with more than one antenna per node, the physical orientations of the antennas were selected in a manner so that the transmission ranges of two antennas on the same node do not overlap. So nodes with two directional antennas had the antennas diametrically opposite to each other. Similarly nodes with four antennas had two pairs of antennas with antennas in each pair diametrically opposite to each other and the pairs being perpendicular to each other. Hence given the number of antennas per node, the orientation of one antenna determined the orientation of other antennas.

TABLE V

Parameters of sectored antennas.

\begin{tabular}{|l||c|c|c|}
\hline & Case1 & Case2 & Case3 \\
\hline Sector angle $^{\circ}$ & 45 & 60 & 90 \\
\hline Number of sectors & 8 & 6 & 4 \\
\hline
\end{tabular}

We compare the total power to construct a broadcast tree for each type of antenna by varying the parameters. A summary of all the parameters used in the simulations for each type of antenna is given in Table V and Table VI. In these tables, the rows containing design within parenthesis denote that a design for the antenna with those parameters has been provided in Section IV-B. The antennas
TABLE VI

Parameters of array-based antennas.

\begin{tabular}{|l|c|c|c|}
\hline \multicolumn{4}{|c|}{ FOVB } \\
\hline Beam width range $^{\circ}$ & 1 ant/node & 2 ant/node & 4 ant/node \\
\hline 8 to 40 (design) & Yes & Yes & Yes \\
\hline 15 to 75 & Yes & Yes & Yes \\
\hline \hline \multicolumn{4}{|c|}{ AOFB (beamwidth $\left.=14^{\circ}\right)$} \\
\hline Scanning angle ${ }^{\circ}$ & 1 ant/node & 2 ant/node & 4 ant/node \\
\hline 30 to 150 (design) & Yes & Yes & No \\
\hline 10 to 170 & Yes & Yes & No \\
\hline \hline \multicolumn{4}{|c|}{${\left.\text { AOVB (beamwidth range is }\left[10^{\circ}, 30^{\circ}\right]\right)}^{\circ}$} \\
\hline Scanning angle & 1 ant/node & 2 ant/node & 4 ant/node \\
\hline 60 to 120 (design) & Yes & Yes & Yes \\
\hline 30 to 150 & Yes & Yes & No \\
\hline 10 to 170 & Yes & Yes & No \\
\hline
\end{tabular}

with other parameters values can also be designed in a similar way, however, at increased design complexity and cost.

We measured the total transmission power of the broadcast trees constructed. The results are plotted using the notion of normalized tree power [30]. We plot the normalized tree power on the y-axis in a $\log$ scale. Let $p_{i}(m)$ denote the total power of the broadcast tree for a network instance $m$, generated by algorithm $i$. Let $p_{0}$ be the power of the lowest-power broadcast tree among the set of algorithms performed and all network instances (50 in our case). Then the normalized tree power associated with algorithm $i$ and network instance $m$ is defined as $p_{i}^{\prime}(m)=\frac{p_{i}(m)}{p_{0}}$.

\section{Results}

Figure 4 shows the power consumed by the broadcast trees constructed using antennas of various types and Table VII shows the percentage of network topologies for which network partition occurs under each antenna type and number.

TABLE VII

Percentage of network topologies with network partitioning for each antenna type/parameter.

\begin{tabular}{|l||c|c|c|c|c|}
\hline \multicolumn{1}{|c||}{} & \multicolumn{5}{c|}{ Number of nodes } \\
\cline { 2 - 6 } Antennas & 10 & 20 & 50 & 100 & 200 \\
\hline \hline BIP & 0 & 0 & 0 & 0 & 0 \\
\hline Sectored(45) & 0 & 0 & 0 & 0 & 0 \\
Sectored(60) & 0 & 0 & 0 & 0 & 0 \\
Sectored(90) & 0 & 0 & 0 & 0 & 0 \\
\hline FOVB-1-(8-40) & 96 & 92 & 44 & 18 & 28 \\
FOVB-1-(15-75) & 80 & 44 & 22 & 12 & 16 \\
FOVB-2-(8-40) & 66 & 22 & 4 & 0 & 0 \\
FOVB-2-(15-75) & 14 & 2 & 0 & 0 & 0 \\
FOVB-4-(8-40) & 0 & 0 & 0 & 0 & 0 \\
FOVB-4-(15-75) & 0 & 0 & 0 & 0 & 0 \\
\hline AOFB-1-(30-150)(14) & 88 & 62 & 30 & 2 & 12 \\
AOFB-1-(10-170)(14) & 72 & 64 & 14 & 0 & 4 \\
AOFB-2-(30-150)(14) & 0 & 0 & 0 & 0 & 0 \\
AOFB-2-(10-170)(14) & 0 & 0 & 0 & 0 & 0 \\
\hline AOVB-1-(60-120)(10-30) & 86 & 56 & 22 & 8 & 16 \\
AOVB-1-(30-150)(10-30) & 62 & 26 & 14 & 2 & 6 \\
AOVB-1-(10-170)(10-30) & 38 & 14 & 8 & 0 & 2 \\
AOVB-2-(60-120)(10-30) & 14 & 0 & 0 & 0 & 0 \\
AOVB-2-(30-150)(10-30) & 0 & 0 & 0 & 0 & 0 \\
AOVB-2-(10-170)(10-30) & 0 & 0 & 0 & 0 & 0 \\
AOVB-4-(60-120)(10-30) & 0 & 0 & 0 & 0 & 0 \\
\hline
\end{tabular}

Figure 4(a) shows the normalized tree power using sectored antennas. We can see that sectored antennas easily outperform 


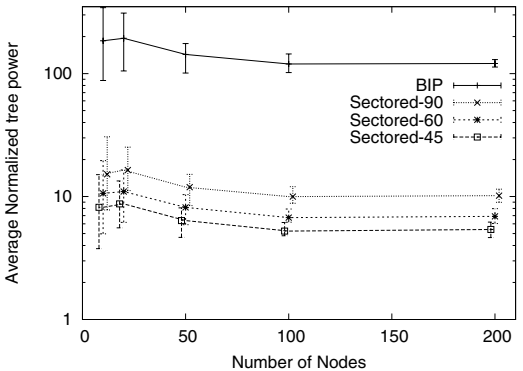

(a) Normalized tree power using sectored antennas with $45^{\circ}, 60^{\circ}$ and $90^{\circ}$ sectors.

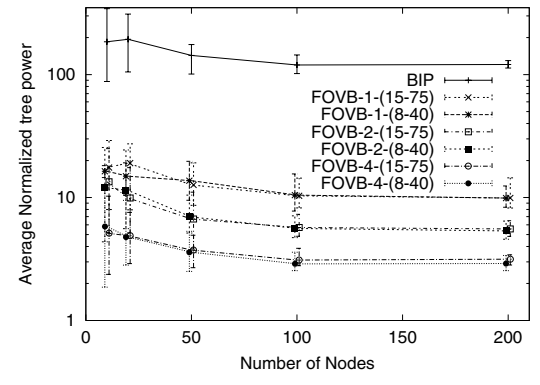

(b) Normalized tree power using FOVB antennas.

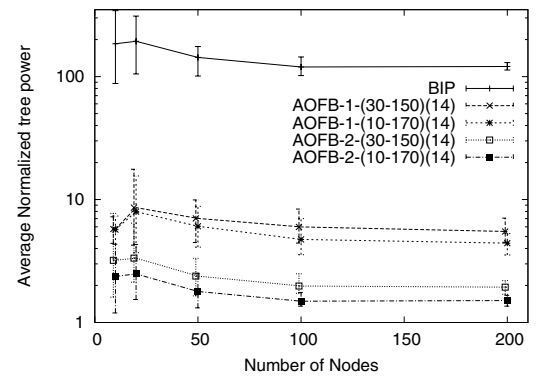

(c) Normalized tree power using AOFB antennas.

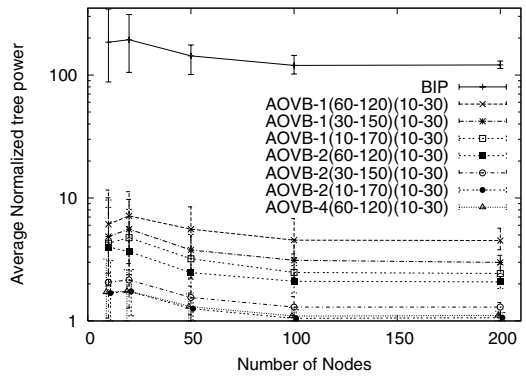

(d) Normalized tree power using AOVB antennas.

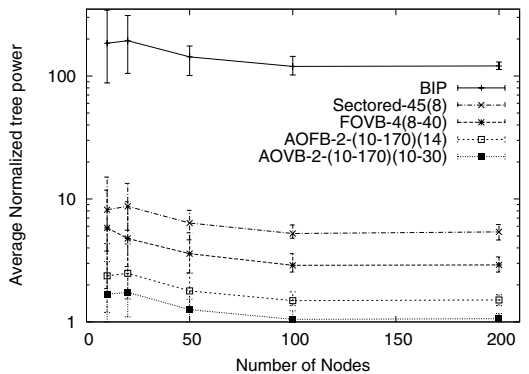

(e) Normalized tree power using all types of antennas.

Fig. 4. Performance of broadcast trees using antennas of various types.

omnidirectional antennas. It is intuitive because we can switch off those sectors which do not reach any node and thus save energy. The power further decreases as we increase the number of sectors thereby decreasing the angle of each sector. Table VIII shows the average number of sectors transmitting per node. We can see that the average number increases as we decrease the sector angle, but the advantage obtained from using smaller sectors outweights this factor. Hence antennas with smaller sectors win comprehensively.

TABLE VIII

Average number of sectors used per node.

\begin{tabular}{|l||c|c|c|}
\hline \# Nodes & $45^{\circ}$ Sectors & $60^{\circ}$ Sectors & $90^{\circ}$ Sectors \\
\hline 10 & 1.7 & 1.6 & 1.5 \\
\hline 20 & 1.6 & 1.5 & 1.5 \\
\hline 50 & 1.5 & 1.5 & 1.4 \\
\hline 100 & 1.5 & 1.5 & 1.4 \\
\hline 200 & 1.5 & 1.5 & 1.4 \\
\hline
\end{tabular}

Figure 4(b) shows the normalized tree power for FOVB antennas. Table VII shows that for one and two antenna cases the network partitioning is high. With four antennas per node there is no network partitioning. Since the four antennas are oriented $90^{\circ}$ apart, they will cover the maximum possible area around a node. We observe that antennas with beamwidth in range $\left[8^{\circ}, 40^{\circ}\right]$ usually win over the antennas with beamwidth in range $\left[15^{\circ}, 75^{\circ}\right]$, although the margin is very small. The reason is a smaller minimum beamwidth of $8^{\circ}$ as compared to $15^{\circ}$ for the other. So, in many cases nodes transmit with $15^{\circ}$ beamwidth even though a smaller beamwidth would have sufficed. However, this advantage comes at a risk. Since the range of beamwidth is small, i.e., the maximum is only $40^{\circ}$ as compared to $75^{\circ}$ for the other, the coverage is less. Consequently, if there are not enough antennas per node then there are more chances of network partitioning for the $\left[8^{\circ}, 40^{\circ}\right]$ beamwidth antennas as shown in Table VII. So it is beneficial to have antennas with smaller beamwidth if there are large number of antennas per node but if the number of antennas is limited then the conservative approach would be to go with larger beamwidth range. For antennas with a fixed beamwidth range, the performance improves as the number of antennas per node increases. This is simply because more antennas per node reduces the chance of antenna exhaustion at a particular node and a nearby node getting connected to a faraway node in the broadcast tree. As expected, omnidirectional antennas lose out to these antennas.

Figure 4(c) shows the performance results for AOFB antennas. The parameters used for the simulations are shown in Table VI. Table VII shows that for one antenna case the network partitioning is high. This is because one antenna is not able to cover the entire $360^{\circ}$ around a node. Also, like in the case with FOVB antennas, increasing the number of antennas per node improves the performance. However, here two antennas suffice because the scanning angle is large enough to cover the entire $360^{\circ}$ around a node with two diametrically opposite antennas. It is interesting to note that as the scanning angle is increased the performance improves, i.e., the broadcast energy values are much less with a scanning angle of $160^{\circ}$ than with a scanning angle of $120^{\circ}$. To explain this, consider the following situation where each node has two antennas. Now let's say that a node $a$ can scan from angle $30^{\circ}$ to $150^{\circ}$ from the positive $\mathrm{x}$-axis using one of its antennas and $210^{\circ}$ to $330^{\circ}$ using its other antenna. If there is a node 
$b$ situated very near $a$ at a position such that the vector from $a$ to $b$ makes an angle of 160 from the $\mathrm{x}$-axis, then none of the antennas of $a$ can reach $b$ and in the final tree $b$ may be a downstream node of a far-off node. However, if the scanning angle was $160^{\circ}$ then $a$ would have easily reached $b$ thereby saving energy. As the scanning angle decreases such cases become more prominent leading to a degradation in performance. It is important to note that arbitrarily increasing the scanning angle is not practical. As expected, omnidirectional antennas lose out to these antennas.

Figure 4(d) shows the performance results for AOVB antennas. The parameters used for the simulations are shown in Table VI. Table VII shows that for antennas with a scanning angle of $120^{\circ}$ or more it suffices to have two antennas to prevent network partitioning so we do not simulate with four antennas having a scanning angle of $120^{\circ}$ or more. However, for a scanning angle of $60^{\circ}$ four antennas are needed. As in the previous cases, as the number of antennas per node increases, the chance of network partitioning decreases and the performance improves. Just as in the case with AOFB antennas, for a given number of antennas per node the performance improves as the scanning angle increases. Again, as expected omnidirectional antennas lose out to these antennas.

Finally, Figure 4(e) compares different types of antennas, using the best parameters from each type. Clearly, the case with two AOVB antennas per node with a scanning angle of $160^{\circ}$ each wins. It is followed by the case with two AOFB antennas per node with a scanning angle of $160^{\circ}$ each. Next is the case with four FOVB antennas per node with a beamwidth range from $8^{\circ}$ to $40^{\circ}$ each. Then comes the case with sectored antennas with $45^{\circ}$ sectors. Last is the case with omnidirectional antennas. The following observations can be made. (i) In general, the performance improves as the antennas are made more flexible, i.e., antennas are allowed to change more parameters (i.e., beamwidth and orientation). (ii) Adjustable orientation results in a larger improvement on the tree power than variable beamwidth. (iii) Increasing the number of antennas per node reduces the tree power. However, increasing the number of antennas arbitrarily may not guarantee better performance. This is shown in Figure 4(d) where using two AOVB antennas per node with $160^{\circ}$ scanning angle yields lower tree power than using four AOVB antennas per node with $60^{\circ}$ scanning angle. So, it suffices to have the minimum number of antennas that will ensure network connectivity. (iv) Finally, for AOVB and AOFB antennas, the scanning angle is the dominant factor in reducing the broadcast energy, i.e., it is better to have fewer antennas with a larger scanning angle than more antennas with a smaller scanning angle as long as it covers $360^{\circ}$ around a node.

\section{CONCLUSION}

We have performed a comprehensive study of the minimumenergy broadcast problem using practical directional antennas. First, we proposed the RF design and implementation of antenna arraybased smart antennas with varying degrees of beam orientation and beamwidth. Our design suggested the set of practical parameters of antennas under each model. We then studied the complexity of the minimum-energy broadcast problem under each of the antenna models and presented heuristic algorithms under different directional antenna models. Finally, we experimentally compared the energy efficiency of the heuristics algorithms using different types of directional antennas. Our simulation results have shown the relative improvement on the broadcast tree power due to different directional antennas design parameters, such as the orientation, the beamwidth, and the number of antennas per node.
The proposed array-based directional antennas are being implemented and will be deployed in a mesh network testbed that we have deployed. We plan to conduct experiments to validate the heuristic algorithms proposed in this paper and measure the impacts of obstacles on signal propagation.

\section{ACKNOWLEDGMENTS}

This work was supported in part by NSF grant ANI-0338856. Any opinions, findings, and conclusions or recommendations expressed in this material are those of the authors and do not necessarily reflect the views of the National Science Foundation.

\section{REFERENCES}

[1] C. Balanis. Antenna Theory, Analysis and Design. John Wiley \& Sons, Inc., 1997.

[2] M. Cagalj, J.-P. Hubaux, and C. Enz. Minimum-energy broadcast in allwireless networks: Np-completeness and distribution. In Proc. of ACM MobiCom, September 2002.

[3] S. Chandran. Adaptive Antenna Arrays, Trends and Applications. Springer, 2004.

[4] J.-H. Chang and L. Tassiulas. Energy conserving routing in wireless ad-hoc networks. In Proc. of IEEE INFOCOM, March 2000.

[5] B. Chen, K. Jamieson, H. Balakrishnan, and R. Morris. Span: An energy-efficient coordination algorithm for topology maintenance in ad hoc wireless networks. In 7th ACM MobiCom, July 2001.

[6] T. H. Cormen, C. E. Leiserson, and R. L. Rivest. Introduction to Algorithms. The MIT Press, Cambridge, MA, 1990.

[7] C. L. Dolph. A current distribution for broadside arrays which optimizes the relationship between beamwidth and sidelobe level,. Proc. IRE, 34(6):335-348, 1946.

[8] O. Egecioglu and T. F. Gonzalez. Minimum-energy broadcast in simple graphs with limited node power. In Proc. of IASTED Int. Conf. on Parallel and Distributed Computing and Systems (PDCS), August 2001.

[9] R. S. Elliott. Antenna Theory and Design. Prentice-Hall, Inc., 1981.

[10] A. Farago and V. R. Syrotiuk. Algorithmic problems in power-controlled ad hoc networks. In Proc. of the 14th Int. Conf. on Parallel and Distributed Computing Systems (PDCS), August 2001.

[11] M. Garey and D. Johnson. Computers and Intractability: A Guide to the Theory of NO-Completeness. W. H. Freeman and Company, 1979.

[12] S. Guo and O. Yang. Antenna orientation optimization for minimumenergy multicast tree construction in wireless ad hoc networks with directional antennas. In Proc. of IEEE/ACM Mobihoc, May 2004.

[13] R. C. Hansen. Phased Array Antennas. John Wiley \& Sons Inc., 1997.

[14] Y. Hou, Y. Shi, , H. Sherali, and J. Wieselthier. Online lifetime-centric multicast routing for wireless ad hoc networks with directional antennas. In Proc. of IEEE INFOCOM, March 2005.

[15] D. B. Johnson and D. A. Maltz. Dynamic Source Routing in Ad Hoc Wireless Networks. Kluwer Academic, 1996.

[16] I. Kang and R. Poovendran. S-GPBE: A power-efficient broadcast routing algorithm using sectored antenna. In Proc. of IASTED WOC'O3, July 2003

[17] X.-Y. Li, W.-Z. Song, and W. Wang. A unified energy-efficient topology for unicast and broadcast. In Proc. of ACM Mobicom, August 2005.

[18] W. Liang. Constructing Minimum-Energy Broadcast Trees In Wireless Ad Hoc Networks. In Proc. of ACM MobiHoc, June 2002.

[19] G. Lin and G. Noubir. Energy efficient broadcast with sectored antennas in wireless ad hoc networks. In Proc. of IASTED WOC'O2, July 2002.

[20] Y. Lu, D. Peroulis, and L. Katehi. An X-band Tunable Class E Power Amplifier. to appear in IEEE Transactions on Microwave Theory and Techniques.

[21] R. J. Mailloux. Phased Array Antenna Handbook. Artech House, 1994.

[22] A. Orda and B.-A. Yassour. Maximum-lifetime routing algorithms for networks with omnidirectional and directional antennas. In Proc. of IEEE/ACM Mobihoc, March 2005.

[23] C. E. Perkins and E. M. Royer. Ad hoc on-demand distance vector routing. In Proc. of IEEE WMCSA, February 1999.

[24] D. Peroulis, , S. Pacheco, , K. Sarabandi, and L. Katehi. Electromechanical Considerations in Developing Low-Voltage RF MEMS Switches. IEEE Transactions on Microwave Theory and Techniques, 51(1):259 270, January 2003.

[25] Radant MEMS, 2003. http://www.radantmems.com/radantmems/index.html. 
[26] G. Rebeiz. RF MEMS Theory, Design, and Technology. John Wiley \& Sons, Inc., New York, NY, 2003.

[27] H. L. V. Trees. Optimum Array Processing (Detection, Estimation, and Modulation Theory, Part IV. John Wiley \& Sons, Inc., 2002.

[28] G. Tsoulos. Adaptve Antennas for Wireless Communications. IEEE Press, 2000.

[29] P. Wan, G. Calinescu, X. Li, and O. Frieder. Minimum-energy broadcasting in static ad hoc wireless networks. Wireless Networks, 8:607-617., 2002. Conference version at IEEE INFOCOM 2001.

[30] J. E. Wieselthier, G. D. Nguyen, and A. Ephremides. On the Construction of Energy-Efficient Broadcast and Multicast Trees in Wireless Networks. In Proc. of IEEE INFOCOM, March 2000.

[31] J. E. Wieselthier, G. D. Nguyen, and A. Ephremides. Energy-efficient broadcast and multicast trees in wireless networks. Mob. Netw. Appl., 7(6):481-492, 2002.

[32] J. E. Wieselthier, G. D. Nguyen, and A. Ephremides. Energy-Limited Wireless Networking with Directional Antennas: The Case of SessionBased Multicasting. In Proc. of IEEE INFOCOM, June 2002.

[33] Y. Xu, J. Heidemann, and D. Estrin. Adaptive energy-conserving routing for multihop ad hoc networks. Research Report 527, USC/Information Sciences Institute, October 2000.

[34] Y. Xu, J. Heidemann, and D. Estrin. Geography-informed energy conservation for ad hoc routing. In 7th ACM MobiCom, July 2001.

[35] R. Zhang and R. Kravets. On-demand power management for ad hoc networks. In Proc. of IEEE INFOCOM, March 2003.

\section{APPENDIX}

In this section, we include the simulated radiation patterns for array based antenna designs.

Theorem 2: The problem of finding a minimum-energy broadcast tree with each node having fixed orientation and fixed beamwidth antenna is NP complete.

We prove that the above problem is NP-complete. To show this, we reduce the Set Cover problem [11] which is known to be NPcomplete.

INSTANCE: Given a set of subsets $S=\left\{S_{1}, S_{2}, \cdots, S_{m}\right\}$ of the universal set $U$ with a weight $w\left(S_{i}\right)$ associated with each $S_{i}$.

QUESTION: Is there subset $T=\left\{T_{1}, T_{2}, \cdots, T_{|T|}\right\}$ of $S$ such that $\cup_{1}^{|T|} T_{i}=U$ and $\sum w\left(T_{i}\right) \leq B$.

Given an instance of the above Set Cover problem, we perform the reduction as follows. We create another graph $G^{\prime}=\left(V^{\prime}, E^{\prime}\right)$. There is a vertex $v^{\prime} \in V^{\prime}$ for each element $s_{i k} \in S_{i}$. We denote it as $v_{s_{i k}}$. There is a vertex $v^{\prime} \in V^{\prime}$ for each $S_{i} \in S$. We denote it as $v_{S_{i}}$. There is a special node $v^{\prime} \in V^{\prime}$ which we denote as $v_{\text {root }}$. Thus all $v_{s_{i k}}$, $v_{S_{i}}$ and $v_{\text {root }}$ make up $V^{\prime}$. There is an edge $e^{\prime}=\left(u^{\prime}, v^{\prime}\right) \in E^{\prime}$ if $u^{\prime}=v_{S_{i}}$ and $v^{\prime}=v_{s_{i k}}$ for some $i$ and $k$. The cost of each such edge $\left(v_{S_{i}}, v_{s_{i k}}\right)$ is $w\left(S_{i}\right)$. Also, each edge $e^{\prime}=\left(u^{\prime}, v^{\prime}\right) \in E^{\prime}, \forall v^{\prime}=v_{S_{i}}$ and $u^{\prime}=v_{\text {root }}$ has a cost $c_{u^{\prime} v^{\prime}}=0$. The interpretation is as follows. For each element $S_{i} \in S$ we have a transmitting node of the type $v_{S_{i}}$ which can reach nodes $v_{s_{i k}}$ when it transmits at power $w\left(S_{i}\right)$ and nobody when it transmits at a lesser power. Also, we have a root node $v_{\text {root }}$ which can reach all the nodes $v_{S_{i}}$ at zero cost. So the above Set Cover problem is equivalent to finding a minimum-energy broadcast tree rooted at $v_{\text {root }}$.

Proof: We now prove that the above problem is NP-complete. Clearly the above problem is in NP because one can guess the powers of the antennas and check in polynomial time whether the total broadcast power is less than or equal to $B$ and whether all the nodes are reached.

We continue by proving that a solution for the Set Cover problem implies a solution for the reduced problem and vice versa.

Suppose that we have a solution for the Set Cover problem. This implies that we know the set $T \subseteq S$. Let $T=\left\{T_{1}, T_{2}, \cdots, T_{b}\right\}$. Also, $\sum w_{T_{i}} \leq B$. We claim that the minimum-energy broadcast solution is as follows. Choose node $v_{S_{i}}$ to transmit at $w\left(S_{i}\right)$ if
$S_{i} \in T$. This implies that all nodes $v_{s_{i k}}$ are reached. Also, allow the node $v_{\text {root }}$ to be in the minimum-energy broadcast tree as this can reach $v_{S_{i}}, \forall i$ at zero cost. Clearly, the total cost of this tree is $\sum w_{T_{i}}$ which is less than or equal to $B$. Since $v_{\text {root }}$ can reach node $v_{S_{i}}, \forall i$, all such nodes are covered. Also, the Set Cover problem guarantees that $\cup_{1}^{|T|} T_{i}=U$ which implies that all the nodes in $V^{\prime}$ in the reduced problem are covered. This is because the only other nodes that the reduced problem contains in addition to the ones in the Set Cover problem are $v_{\text {root }}$ and $v_{S_{i}}, \forall i$. Since, $v_{\text {root }}$ reaches $v_{S_{i}}, \forall i$ by transmitting at zero power, all the nodes are covered. Clearly, the total broadcast power is equal to $\sum w_{T_{i}}$ which is less than or equal to $B$.

Now, we prove that given a solution to the minimum-energy broadcast problem we have a solution to the Set Cover problem. Let's say we have a set of nodes $V_{t} \subseteq V^{\prime}$ transmitting at power $p\left(v_{t}\right), \forall v_{t} \in V_{t}$ and $\sum p\left(v_{t}\right) \leq B$. $v_{\text {root }} \in V_{t}$ because there is no other way to reach nodes $v_{S_{i}}$ for any $i$. We claim that the Set Cover solution is as follows. Choose a solution set $T$ such that $S_{i} \in T$ if and only if $v_{S_{i}} \in V_{t}$ and $p\left(v_{S_{i}}\right) \geq w\left(S_{i}\right)$. Clearly, the total cost $\sum w_{T_{i}} \leq B$, where $T_{i} \in T$ because we have chosen the nodes in such a way that the total broadcast power is less that $B$ and the power of transmission $p\left(v_{t}\right) \geq w\left(S_{i}\right)$. If there exists a node $v_{t} \in V_{t}$ but $v_{t} \neq v_{S_{i}}$ for any $S_{i}$ and $v_{t} \neq v_{\text {root }}$, then node $v_{t}$ can not reach any other node because it is of the form $v_{s_{i k}}$ and according to the reduction such nodes do not have any edge to other nodes. Also, $\cup_{1}^{|T|} T_{i}=U$ where $T_{i} \in T$ because the minimum-energy broadcast solution guarantees that all nodes are covered. This completes the proof.

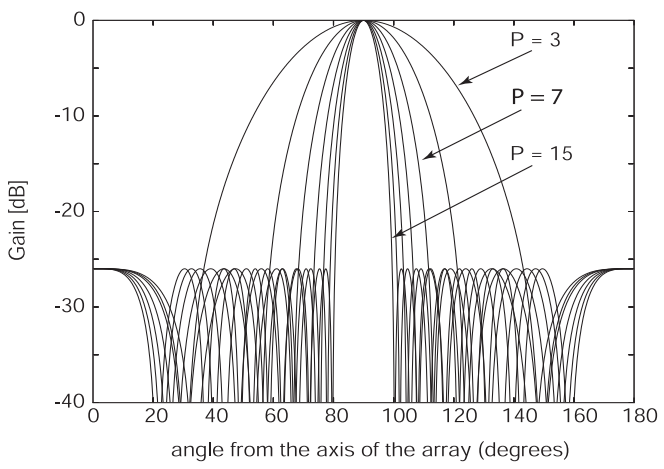

Fig. 5. Simulated radiation pattern for a fixed-orientation adjustablebeamwidth antenna. The highest and lowest beamwidths are provided with 3 and 15 elements in the array respectively. The beamwidths for all designs are summarized in Table I. 


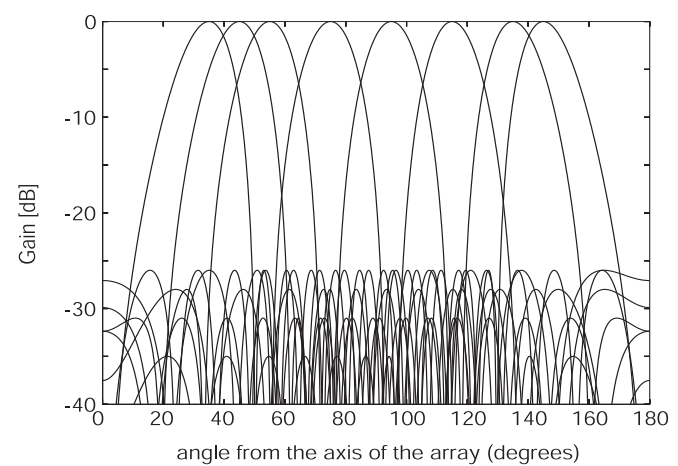

Fig. 6. Scanning of the proposed antenna array at various angles. Even for such wide scanning the beamwidth remains constant. The details are summarized in Table II.

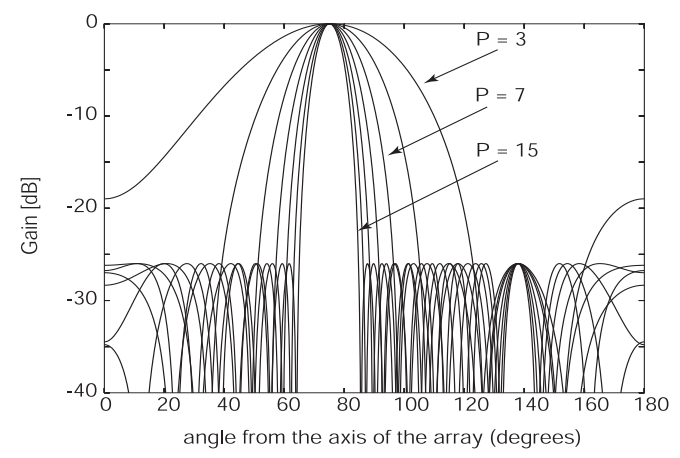

(a) Scanning angle $=75$ degrees

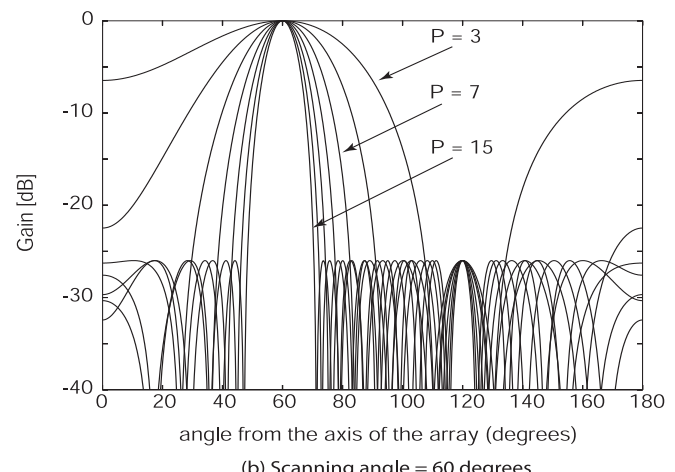

Fig. 7. Adjustable beamwidths for two different scanning angles. The details are summarized in Table III. 